\title{
Study on the Application of Electric Field to Giant Vesicles Comprised of 1,2-Dilauroyl-Sn- Glycero-3-Phosphatidylcholine Using Optical Fluorescence Microscopy
}

\author{
Yasmine Miguel Serafini Micheletto ${ }^{a *}$, Nádya Pesce da Silveira ${ }^{a}$, Alexandre Gonçalves Dal-
}

\author{
Bób $^{b}$, Fernando Carlos Giacomellic, Newton Luiz Dias Filho ${ }^{b}$, Tiago Elias Allievi Frizon ${ }^{b}$,
}

Carlos Manuel Marques ${ }^{d}$, André Pierre Schroder ${ }^{d}$

\author{
${ }^{a}$ Graduate Program in Chemistry, Institute of Chemistry, Federal University of Rio Grande do Sul State, \\ Porto Alegre, Brazil. \\ ${ }^{b}$ University of the Extreme South of Santa Catarina State - UNESC, 88806-000 Criciúma, SC, Brazil \\ c Universidade Federal do ABC - UFABC, 09210-170, Santo André, SP, Brazil \\ ${ }^{d}$ Charles Sadron Institute, University of Strasbourg, UPR22-CNRS, 23 rue du Loess, \\ 67034, Strasbourg, France
}

Received: September 20, 2016; Revised: February 22, 2017; Accepted: April 05, 2017.

\begin{abstract}
The influence of alternating electric field (AC) in the structure and dynamics of giant unilamellar vesicles (GUVs) comprised of 1,2-dilauroyl-sn-glycero-3-phosphatidylcholine (DLPC) is reported. The investigations were conducted by using optical fluorescence microscopy as the method of analysis. The lipid membrane of the DLPC GUVs at the fluid phase can be deformed and they migrate towards the electrodes under AC electric field. Nevertheless, membrane disruption or vesicle fusion was never noticed. The addition of concentrated glucose solution influences the osmotic pressure of the system leading to the formation of filaments at the outer region of the GUVs. These long flexible cylinders do not retract spontaneously. However, the application of AC electric field $(20 \mathrm{~V} / \mathrm{mm}, 20 \mathrm{~Hz})$ enables the filaments to be retracted back to the GUVs membrane at a calculated speed of $0.18 \mu \mathrm{m} . \mathrm{s}^{-1}$
\end{abstract}

Keywords: Electric field, GUVs, DLPC, Fluorescence microscopy

\section{Introduction}

Perturbations of lipid systems via the application of external electric fields can provide informations on their structural features and dynamic properties. The exposure of cell membranes to alternating electric fields may lead to a variety of responses, such as for instance, cell deformation and effects on the mobility and cell shape ${ }^{1-6}$ Along these lines, giant unilamellar vesicles (GUVs) have been extensively investigated as a model for biological membranes since their dimension are compared to living cells $(5-50 \mu \mathrm{m})$. Technically, they can be easily observed by using an optical microscope enabling investigations of the membrane behavior under electric field. ${ }^{7-11}$

Indeed, it has been already reported that GUVs behavior under AC (alternating current) and DC (direct or continuous current) is different. The application of AC electric field induces stationary deformations in the vesicles while intense electric fields (pulsed DC) can cause a significant increase in the electrical conductivity and permeability of cell membranes such as in the cases of electroporation and electropermeabilization. ${ }^{1,2,12-16}$ The spherical vesicles under $\mathrm{AC}$ assume an ellipsoidal shapes and the degree of deformation depends on several parameters including electric field strength and frequency. ${ }^{2}$ The application of AC electric fields can also be used to induce vesicle poration. ${ }^{17}$

\footnotetext{
*e-mail: myne83@hotmail.com
}

Taking into account the above mentioned knowledge, herein it is reported the influence of external AC electric field on the dynamic and structure of GUVs produced from 1,2-dilauroyl-sn-glycero-3-phosphatidylcholine (DLPC) by using optical fluorescence microscopy. The motivation was essentially to evaluate the influence of AC electric field in fully saturated hydrocarbon chains as present in DLPC.

\section{Experimental procedures}

\subsection{Samples and Chemicals}

The lipid 1,2-dilauroyl-sn-glycero-3-phosphatidylcholine (DLPC, $>99 \%$ ) and the fluorophore 1,2-dioleoyl-sn-glycero3-phosphatidylethanolamine- $\mathrm{N}$-(lissamine rhodamine $\mathrm{B}$ sulfonyl) (ammonia salt) - (rhodamine-DOPE, > 99\%), in chloroform, were obtained from Avanti Polar Lipids. The cell for the application of the electric field was purchased from Eppendorf.

\subsection{Formation of the Giant Unilamellar Vesicles (GUVs)}

The giant unilamellar vesicles (GUVs) were grown following the electroformation method described by Angelova et al. ${ }^{18}$ Accordingly, $10 \mu \mathrm{L}$ of a $1 \mathrm{mg} . \mathrm{mL}^{-1}$ lipid chloroform 
solution containing a small fraction of fluorescent lipid (ca. $0.5 \% \mathrm{~mol} / \mathrm{mol}$ ) were spread gently with a syringe onto the conductive side of two indium tin oxide (ITO) coated glass slides (PGO, Iserlohn, Germany). Chloroform was evaporated under vacuum for at least $30 \mathrm{~min}$. The two ITO slides were then positioned in order to form a $2 \mathrm{~mm}$-thick electroformation growing chamber (Teflon spacer) with conductive sides facing each other. The chamber was filled with $0.2 \mathrm{M}$ sucrose solution and vesicle growth was achieved by applying an $\mathrm{AC}$ voltage $(2 \mathrm{~V} / \mathrm{mm}$ and $10 \mathrm{~Hz})$ for $3 \mathrm{~h}$. The GUVs were then transferred to a vial and diluted (ca. 50 $\%$ ) in $0.2 \mathrm{M}$ glucose solution. Due to differences between the densities of the inner sucrose and outer glucose+sucrose solutions, the GUVs quickly sediment under gravity. The osmolarities of the sucrose and glucose solutions were previously finely matched using a cryoscopic osmometer (Osmomat 030, Gonotec, Berlin, Germany).

\subsection{Electric Field Setup}

The simultaneous electric field application and optical fluorescence microscopy observations were performed by using the cell setup portrayed in Figure 1. The Teflon cell contains a glass sheet at the lower part allowing the visualization under an optical microscope and two $0.09 \mathrm{~mm}$ diameter electrodes separated by $0.5 \mathrm{~mm}$ located between the lower glass and the upper opening of the cell as indicated by the arrows in Figure 1.

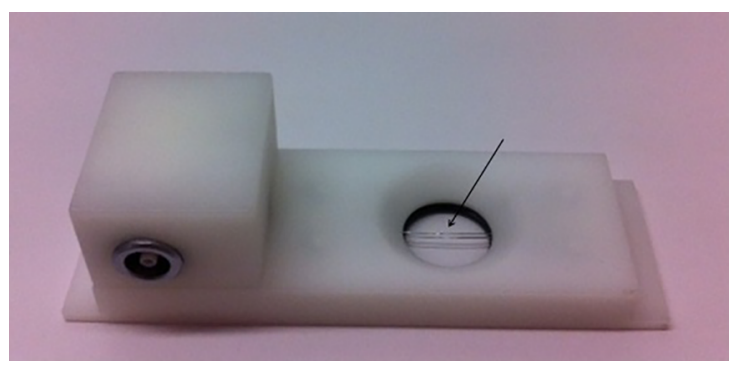

Figure 1. Digital photograph of the cell used for the application of the electric field during the optical fluorescence microscopy.

The long distance between the electrodes as compared to their diameter ensures a homogeneous distribution of the field in the space between the electrodes..$^{19}$ The cell volume is approximately $0.8 \mathrm{~mL}$. The apparatus was previously connected to the microscope and subsequently an adjustable voltage source $(1 \mathrm{~V}-20 \mathrm{~V}, 1 \mathrm{~Hz}-1 \mathrm{MHz})$ was employed to generate $\mathrm{AC}$ electric fields.

\subsection{Optical fluorescence microscopy}

The GUVs were previously observed in the absence of electric field using an inverted microscope (TE 2000, Nikon, Japan) equipped with a 60X objective lens (1.2 NA Plan Apo
DIC objective) immersed in water. The three dimensional (3D) images were obtained using confocal laser scanning microscopy (CLSM) with a Nikon C1 scanner. The images were captured with the EZ-C1 software (Nikon, version 3.50). The fluorescence of the fluorophore rhodamine-DOPE present in the GUVs membranes was detected in confocal mode after excitation at $543 \mathrm{~nm}$ (helium-neon laser, MellesGriot). The images were analyzed using the software Magneto developed at the Charles Sadron Institute. The whole set of data was acquired at $18{ }^{\circ} \mathrm{C}$.

The fluorescence images of the GUVs under the application of electric field were obtained using an inverted microscope (TE2000, Nikon, Japan) equipped with a 40X objective lens because of the thickness of the glass sheet used to build the electric field cell. The 40X objective lens was previously used in phase contrast mode in order to locate the electrodes and vesicles and further switched to fluorescence mode for the application of electric fields. The microscope uses a mercury lamp as light source with excitation filter placed within the illumination path for the selection of the incident wavelength. The fluorescence images were collected using a digital camera (Diagnostic Instruments NDIAG 1800) coupled to the microscope and allowing the acquirement of images and videos.

\section{Results and discussion}

The phase transition temperature $\left(T_{f}\right)$ of DLPC is located at about $-1{ }^{\circ} \mathrm{C}$. Accordingly, the GUVs are in the fluid phase at the temperature of the experiments $\left(18^{\circ} \mathrm{C}\right)$. The vesicles could thus be deformed under external electric field as evidenced in the sequence of images portrayed in Figure 2. The application of the higher electric field $(18 \mathrm{~V} / \mathrm{mm}, 60$ $\mathrm{Hz}$ ) also moves the vesicles towards the electrodes. As the strength of the electric field is lowered, the vesicles have the tendency to move away from the electrodes losing fragments of their membrane (Figure 2b). Application of successive cycles leads to increased vesicle mobility and a reduction in their size and the formation of filaments (Figures 2c-d).

The deformation is explained taking into account the fluid phase and the lipid nature of the bilayer. The structural characterization of DLPC, DMPC and DOPC unilamellar vesicles through SAXS measurements evidenced that the hydrophobic region of DOPC is $2 \AA$ thicker than that of DMPC, although the carbon chains of the previous are reasonable bigger (four carbon atoms longer). ${ }^{20}$ Therefore, hydrophobic interactions in DOPC are presumably stronger even though DOPC is not saturated. This behavior probably reflects the molecular weight of the hydrophobic chains and the increasing in London dispersion forces, thereby restricting the deformation of DOPC GUVs under external electric field as compared to DMPC and DLPC. On the other hand, the dimension of the DLPC hydrophobic region is $4.5 \AA$ smaller compared to DMPC, although the latter has only two carbon 
atoms more at each carbon chain. ${ }^{20}$ The available area per chain at the bilayer is also lipid-dependent $\left(72.1 \AA^{2}\right.$ for DOPC, $60.6 \AA^{2}$ for DMPC and $63.2 \AA^{2}$ for DLPC). ${ }^{20}$ Therefore, the degree of deformation observed in DLPC vesicles under external electric field may have its explanation in the weaker hydrophobic interactions (because of the lower number of carbons at each hydrophobic chain), the more voluminous packing of the lipid chains as compared to DMPC, as well as the fluid state allowing more flexibility to the membrane.
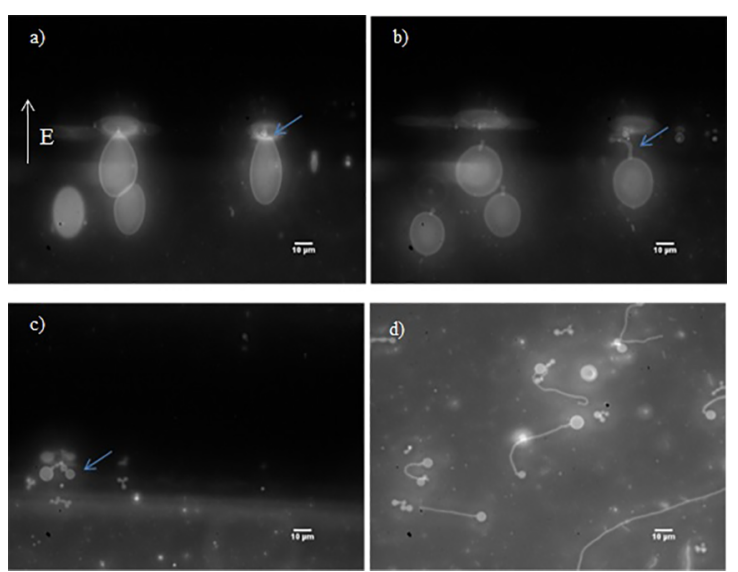

Figure 2. Sequence of optical fluorescence microscopy images of the GUVs produced from DLPC and rhodamine with the application of: (a) and (c) $18 \mathrm{~V} / \mathrm{mm}$ and $60 \mathrm{~Hz}$; (b) and (d) $4 \mathrm{~V} / \mathrm{mm}$ and $60 \mathrm{~Hz}$.

The addition of $40 \mu \mathrm{L} 1 \mathrm{M}$ glucose solution leads to the formation of two different types of filaments when the system is under electric field. The filaments remain connected to the GUVs membrane. One type of filament is thicker (average diameter $\sim 2 \mu \mathrm{m}$ ) and in less than $1 \mathrm{~min}$ they return to make part of the membrane again (Figure 3). Additionally, when the applied electric field is $10 \mathrm{~V} / \mathrm{mm}$, these filaments instantly return to the vesicles (not shown).
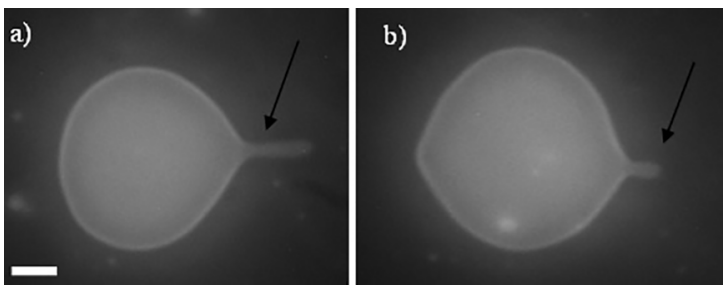

Figure 3. Sequence of optical fluorescence microscopy images of GUVs comprised of DLPC and $0.5 \%$ of rhodamine: (a) immediately after the addition of 3 drops of concentrated glucose and (b) natural return of the filament to the vesicle. Bar $=5 \mu \mathrm{m}$.

The DLPC vesicles also become smaller as glucose is progressively added to the system (Figure 4). The arrows depicted in Figure 4 points the formation of smaller vesicles produced by the division of the larger assemblies caused by the osmotic shrinkage induced by the concentration gradient. The increase in solute concentration results in water influx towards the outer part of the vesicles in order to balance the concentration of both compartments thereby leading to and imbalanced surface/volume ratio in the vesicles and ultimately resulting in the formation of smaller assemblies.

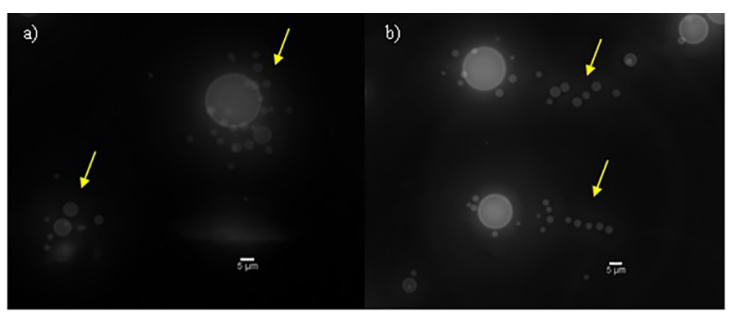

Figure 4. Optical fluorescence microscopy images of GUVs comprised of DLPC and $0.5 \%$ of rhodamine after successive additions of $1 \mathrm{M}$ glucose.

The Figure 5 depicts the vesicles after the addition of concentrated glucose solution. The GUVs depicted in Figure 5a were observed in the absence of electric field and after the addition of the concentrated glucose solution whereas in Figure $5 \mathrm{~b}$ an electric field of $30 \mathrm{~V} / \mathrm{mm}$ was applied during 10 s (until the elongation of the vesicles) and then it was rapidly reduced to $4 \mathrm{~V} / \mathrm{mm}$ leading to the condition observed in Figures $5 \mathrm{c}-\mathrm{d}$. The formation of filaments as well as smaller vesicles is clearly seen, particularly in Figure $5 \mathrm{~d}$. Indeed, the second type of filament produced with the addition of $40 \mu \mathrm{L}$ $1 \mathrm{M}$ glucose solution is thinner (average diameter $\sim 1 \mu \mathrm{m}$ ). These filaments do not return to the vesicle spontaneously. Several conditions of voltage and frequency were tested until two conditions were established where the filaments return to the vesicle as a result of the application of electric field. For instance, the application of $20 \mathrm{~V} / \mathrm{mm}$ at $20 \mathrm{~Hz}$ enables the filaments to be retracted to the original GUVs. However, the rapid movement of the vesicles in suspension after the addition of glucose hinders the analysis and thus the electric field was applied for 2-5 min after the addition of glucose to the cell. The experiment was carried out with the electric field cell closed.

The Figure 6 shows the average filament length $(\mu \mathrm{m})$ as a function of the total retraction time for $20 \mathrm{GUVs}$ under external electric field $(10 \mathrm{~V}, 20 \mathrm{~Hz})$. The data evidences that the total length of the filaments is linearly dependent of the time they take to be incorporated back to the membrane. For instance, smaller filaments have a shorter retraction time and rapidly return to the vesicle.

Complementary, the Figure 7 shows the filament length as a function of the retraction times. The optical fluorescence microscopy images portray the vesicles at different points of the retraction process. In these data, obviously, the filament is longer at the beginning of the dynamic process becoming progressively shorter as time proceeds. Based on the fitting results, the retraction speed is about $0.18 \mu \mathrm{m} . \mathrm{s}^{-1}$.

Finally, the surface area of the vesicles was also calculated. The value is equal to $1256 \mu \mathrm{m}^{2}$ before the application of 

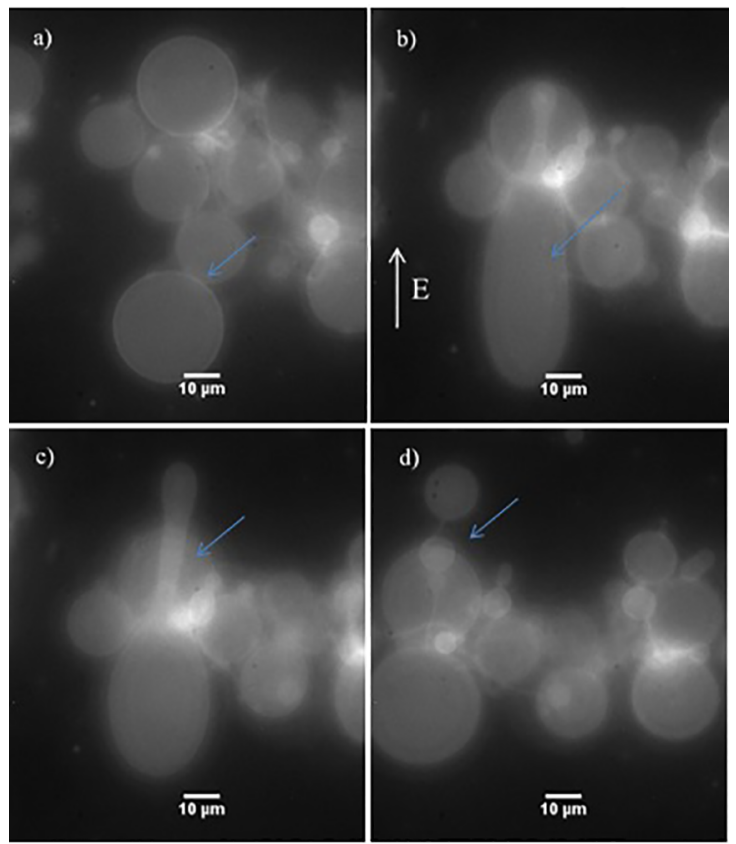

Figure 5. Sequence of optical fluorescence microscopy images of GUVs produced from DLPC and rhodamine: (a) absence of an electric field and after the addition of a concentrated glucose solution, (b) with applied electric field of $30 \mathrm{~V} / \mathrm{mm}, 60 \mathrm{~Hz}$ and (c) and (d) with applied electric field of $4 \mathrm{~V} / \mathrm{mm}, 60 \mathrm{~Hz}$.

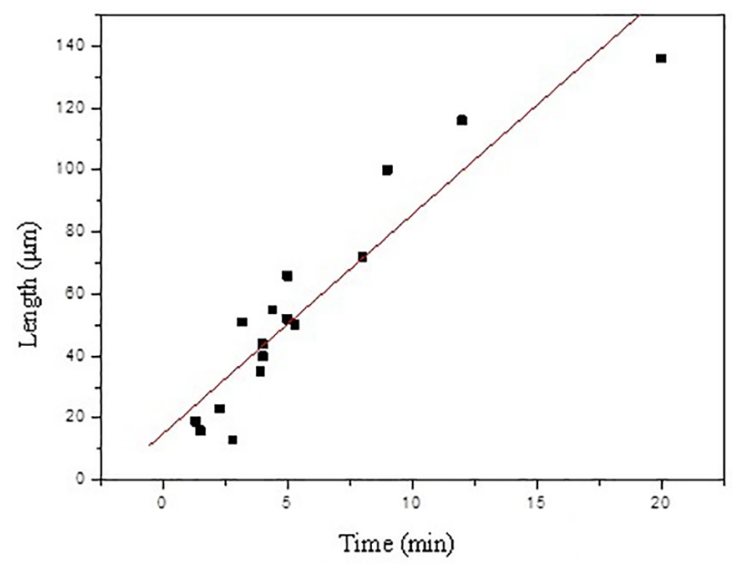

Figure 6. Total length of the filaments as a function of the total retraction time for $20 \mathrm{GUV}$ s of DLPC under external electric field of $10 \mathrm{~V} / \mathrm{mm}, 20 \mathrm{~Hz}$.

electric field whereas after the retraction of the filament the value is $1505 \mu \mathrm{m}^{2}$. The filament area is accordingly $249 \mu \mathrm{m}^{2}$. Presumably, the larger surface area is caused by the retraction of the filament towards the vesicle that they were originated.

\section{Conclusions}

The influence of electric field (AC) on structural features and dynamic properties of GUVs produced from DPLC was investigated using optical fluorescence microscopy. The lipid

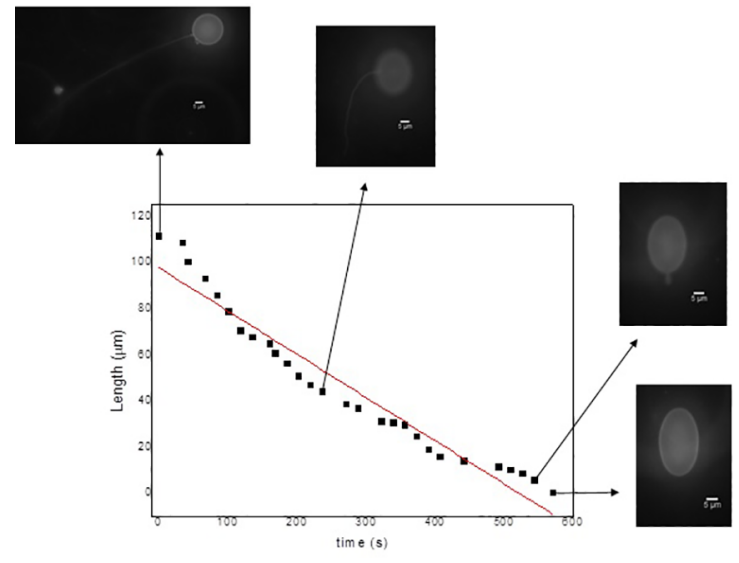

Figure 7. Filament length as a function of the retraction time for a GUV of DLPC under external electric field of $10 \mathrm{~V} / \mathrm{mm}, 20 \mathrm{~Hz}$.

membrane of the GUVs can be deformed by employing electric field cycles (from 2 to $40 \mathrm{~V} / \mathrm{mm}$ at constant frequency). It was also evidenced electrode migration but, on the other hand, membrane permeation was never observed. The reduced number of carbon atoms at the hydrophobic region along to the available area per lipid chain at the bilyaer and the fluid stated of the assemblies can explain the extendend degree of deformation observed by increasing the strength of electric field. The addition of concentrated glucose solution to the system causes osmotic imbalance and enable the formation of filaments at the outer region of the GUVs. These ramifications retract at a speed of $0.18 \mu \mathrm{m} . \mathrm{s}^{-1}$ under external electric field $(20 \mathrm{~V} / \mathrm{mm}, 20 \mathrm{~Hz})$.

\section{Acknowledgments}

The authors wish to thank CAPES (Coordenação de Aperfeiçoamento de Pessoal de Nível Superior) for financial support.

\section{References}

1. Riske KA, Dimova R. Electric Pulses Induce Cylindrical Deformations on Giant Vesicles in Salt Solutions. Biophysical Journal. 2006;91(5):1778-1786.

2. Dimova R, Riske KA, Aranda S, Bezlyepkina N, Knorr RL, Lipowsky R. Giant vesicles in electric fields. Soft Matter. 2007;3(7):817-827.

3. Portet T, Febrer FC, Escoffre JM, Favard C, Rols MP, Dean DS. Visualization of Membrane Loss during the Shrinkage of Giant Vesicles under Electropulsation. Biophysical Journal. 2009;96(10):4109-4121.

4. Friend AW Jr, Finch ED, Schwan HP. Low frequency electric field induced changes in the shape and motility of amoebas. Science. 1975;187(4174):357-359.

5. Engelhardt H, Sackmann E. On the measurement of shear elastic moduli and viscosities of erythrocyte plasma membranes 
by transient deformation in high frequency electric fields. Biophysical Journal. 1988;54(3):495-508.

6. Engelhardt H, Gaub H, Sackmann E. Viscoelastic properties of erythrocyte membranes in high-frequency electric fields. Nature. 1984;307(5949):378-380.

7. Walde P, Cosentino K, Engel H, Stano P. Giant vesicles: preparations and applications. Chembiochem. 2010;11(7):848865 .

8. Wesołowska O, Michalak K, Maniewska J, Hendrich AB. Giant unilamellar vesicles - a perfect tool to visualize phase separation and lipid rafts in model systems. Acta Biochimica Polonica. 2009;56(1):33-39.

9. Campillo CC, Schroder AP, Marques CM, Pépin-Donat B. Composite gel-filled giant vesicles: Membrane homogeneity and mechanical properties. Materials Science and Engineering: C. 2009;29(2):393-397.

10. Antonova K, Vitkova V, Meyer C. Membrane tubulation from giant lipid vesicles in alternating electric fields. Physical Review E. 2016;93(1):012413.

11. Li W, Wang Q, Yang Z, Wang W, Cao Y, Hu N, et al. Luo H. Impacts of electrical parameters on the electroformation of giant vesicles on ITO glass chips. Colloids and Surfaces B: Biointerfaces. 2016;140:560-566.

12. Riske KA, Dimova R. Electro-deformation and poration of giant vesicles viewed with high temporal resolution. Biophysical Journal. 2005;88(2):1143-1155.
13. Haluska CK, Riske KA, Marchi-Artzner V, Lehn JM, Lipowsky R, Dimova R. Time scales of membrane fusion revealed by direct imaging of vesicle fusion with high temporal resolution. Proceedings of the National Academy of Sciences. 2006;103(43):15841-15846.

14. Mauroy C, Rico-Lattes I, Teissié J, Rols MP. Electric Destabilization of Supramolecular Lipid Vesicles Subjected to Fast Electric Pulses. Langmuir. 2015;31(44):12215-12222.

15. Salipante PF, Vlahovska PM. Vesicle deformation in DC electric pulses. Soft Matter. 2014;10(19):3386-3393.

16. McConnell LC, Vlahovska PM, Miksis MJ. Vesicle dynamics in uniform electric fields: squaring and breathing. Soft Matter. 2015;11(24):4840-4846.

17. Harbich W, Helfrich W. Alignment and Opening of Giant Lecithin Vesicles by Electric Fields. Zeitschrift für Naturforschung A. 1979;34(9):1063-1065.

18. Angelova MI, Dimitrov DS. Liposome electroformation. Faraday Discussions of the Chemical Society. 1986;81:303-311.

19. Staykova M, Lipowsky R, Dimova R. Membrane flow patterns in multicomponent giant vesicles induced by alternating electric fields. Soft Matter. 2008;4(11):2168-2171.

20. Kučerka N, Liu Y, Chu N, Petrache HI, Tristram-Nagle S, Nagle JF. Structure of Fully Hydrated Fluid Phase DMPC and DLPC Lipid Bilayers Using X-Ray Scattering from Oriented Multilamellar Arrays and from Unilamellar Vesicles. Biophysical Journal. 2005;88(4):2626-2637. 